\title{
Formación del profesorado a través del programa ERASMUS+: las prácticas educativas innovadoras en los docentes de Reggio Emilia
}

\section{Teacher training through the ERASMUS+ program: innovative educational practices in Reggio Emilia teachers.}

DOI: $10.46932 /$ sfjdv2n2-066

Received in: March 1st, 2021

Accepted in: May 30th, 2021

\author{
Dra. Lidia Blanco Reyes \\ Profesora Asociada \\ Institución: Universidad de Alicante \\ Dirección: Facultad de Educación. Carretera San Vicente del Raspeig s/n, 03690. San Vicente del \\ Raspeig - Alicante \\ E-mail: lidia.blanco@ua.es \\ Dra. Inés Lozano Cabezas \\ Profesora Contratada Doctora \\ Institución: Universidad de Alicante \\ Dirección: Facultad de Educación. Carretera San Vicente del Raspeig s/n, 03690. San Vicente del \\ Raspeig - Alicante \\ E-mail: ines.lozano@ua.es \\ Dr. Marcos Jesús Iglesias Martínez \\ Profesor Titular de Universidad \\ Dirección: Facultad de Educación. Carretera San Vicente del Raspeig s/n, 03690. San Vicente del \\ Raspeig - Alicante \\ E-mail: marcos.iglesias@ua.es
}

\begin{abstract}
RESUMEN
El programa ERASMUS favorece las competencias docentes necesaria para una integración y adaptación de nuevos modelos pedagógicos y estrategias didácticas para el profesorado en activo, permitiendo observar, investigar e indagar estrategias en otros contextos sociales y culturales. El objetivo de esta investigación es conocer y analizar la metodología de las escuelas Reggio Emilia (Italia) mediante una entrevista a los docentes participantes. La observación y el análisis de las experiencias vividas han permitido describir resultados basados en las dimensiones didácticas de los docentes entrevistados. Se identifica un alto compromiso por la educación, así como un trabajo colaborativo entre los docentes para el diseño y desarrollo de las actividades, optando en su mayoría por adaptarse a los intereses del alumnado, una relación directa con las familias y un sistema de evaluación basado en la observación y en la formación. Nuestras conclusiones se orientan a considerar que para mejorar el desarrollo profesional docente es muy significativo conocer y vivenciar directamente estas experiencias, la cuáles son muy relevantes en la formación permanente del profesorado. El programa ERASMUS permite completar la formación de los docentes gracias al conocimiento de otras prácticas educativas innovadoras en otros contextos escolares, claves en cualquier proceso educativo.
\end{abstract}

Palabras clave: Erasmus+, formación del profesorado, investigación cualitativa, práctica educativa. 


\begin{abstract}
The ERASMUS program favors the teaching competences necessary for the integration and adaptation of new pedagogical models and didactic strategies for active teachers, allowing them to observe, investigate and research strategies in other social and cultural contexts. The objective of this research is to know and analyze the methodology of the Reggio Emilia schools (Italy) by means of an interview to the participating teachers. The observation and analysis of the lived experiences have made it possible to describe results based on the didactic dimensions of the teachers interviewed. A high commitment to education is identified, as well as a collaborative work among teachers for the design and development of activities, mostly choosing to adapt to the interests of students, a direct relationship with families and an evaluation system based on observation and training. Our conclusions are oriented to consider that in order to improve the professional development of teachers it is very significant to know and directly experience these experiences, which are very relevant in the continuing education of teachers. The ERASMUS program allows to complete the training of teachers thanks to the knowledge of other innovative educational practices in other school contexts, which are key in any educational process.
\end{abstract}

Key words: Erasmus+, teacher training, qualitative research, educational practice.

\title{
1 INTRODUCCIÓN
}

Como modelo de formación del profesorado el Programa ERASMUS+ (European Commision, 2016 y 2017) se está desarrollando en diferentes países de la Unión Europea, siendo una de las acciones de movilidad que permite formar al profesorado en diversos contextos educativos a través de la observación de distintas experiencias innovadoras que se realizan en distintos centros educativos en el contexto de la educación europea (Carins, Kraklewska, Cuzzocrea y Allaste, 2018). Concretamente sus políticas van encaminadas "al crecimiento, el empleo, la equidad e inclusión” (European Commision, 2016, p.5), como valores que aportan conocimiento al desarrollo profesional docente. En este sentido se trata de una formación que supone la mejora del aprendizaje continuo del profesorado y que, entre otros objetivos, promueve la optimación de sus procesos de enseñanza y de aprendizaje en su práctica educativa. Esta formación propicia adquirir competencias propias de la profesión docente, para integrar y adaptar nuevos modelos didácticos, curriculares y pedagógicos con el propósito implementar estas estrategias educativas en el aula (Dotger, 2015). Asimismo, el programa ERASMUS permite, entre otros aspectos, indagar en esta cultura docente y colaborativa (Flores, 2017; Vaillant, 2016), gracias al contacto con otros profesores de distintos países europeos que comparten sus buenas prácticas. También hay que destacar la observación del trabajo en la comunidad educativa, pues no sólo se trata de una movilidad en un espacio concreto como es el aula, sino que requiere la comunicación y observación asociaciones escolares (familia y escuela) y las redes institucionales del profesorado (Bonell y Ríos, 2016; Opfer y Pedder, 2011).

Estamos de acuerdo con Iglesias, Lozano y Roldán (2018) que la innovación educativa y curricular implica un cambio de las prácticas en las aulas escolares en el que el docente desarrolla su trabajo. Más concretamente la innovación de los docentes intenta evitar la desconexión entre la teoría y la práctica 
(Giner, Iglesias, Lozano y Urrea, 2017). Observar y analizar las situaciones reales de las innovaciones que desarrollan en su práctica docente, suponen un desafío interesante para el desarrollo profesional del profesorado en el Programa ERASMUS+ y, también, en la movilidad estudiantil, ya que según Arroyo, Iglesias y Lozano (2021, p.1486) "esto permite asegurar el acceso, la calidad de la educación y la no discriminación de parte de la Comunidad Educativa [...] son necesarias políticas que aseguren la inclusión del alumnado en todos los procesos de la educación". Las experiencias que se observan y aprenden en el programa ERASMUS+ permite compartir las vivencias didácticas de los centros que se visitan y crear redes docentes con el fin de compartir los beneficios adquiridos (Blanco, Iglesias y Lozano, 2017). En este sentido el modelo Reggio Emilia es una metodología y didáctica que permite al docente experienciar el aprendizaje del alumnado a través de la "denominada pedagogía del ambiente”, centrando su formación en fortalecer las competencias profesionales de los docentes, para responder a los retos de la sociedad multicultural adoptando métodos innovadores de enseñanza y aprendizaje. Nuestro interés de optar por esta región y por resta metodología ha sido para identificar estrategias didácticas que contribuyen muy positivamente a la calidad del aprendizaje de las niñas y los niños de la etapa de Educación Infantil. Destacamos, entre otros, el carácter innovador de este método, ya que los docentes que realizan la movilidad en este programa no sólo les permiten vivenciar las características el entorno, sino también indagar sobre las diferentes situaciones didácticas reales en un contexto educativo de calidad generado por la alta profesionalidad de sus docentes y la dedicación a la educación.

\section{METODOLOGÍA}

Hemos optado por una metodología cualitativa pues nos ha permitido interpretar las auténticas oportunidades que ofrece el programa ERASMUS+ para el profesorado participante, concretamente, acerca de las prácticas educativas que desarrollan en otros contextos educativos de referencia (Atkins y Wallace, 2012), con el propósito de implementar los métodos innovadores en los centros escolares (Dwyer y Emerald, 2017; Goodson, Antikainen, Sikes, y Andrews, 2016). En este sentido las cuestiones de investigación que se han planteado para esta comunicación son los siguientes:

1. ¿Qué elementos curriculares y didácticos son observados como prácticas educativas innovadoras en los docentes de Reggio Emilia?

2. ¿Qué satisfacciones reportan al docente en su quehacer diario gracias a esta metodología?

3. ¿Qué aspectos se destacan del trabajo colaborativo entre docentes y familias en la escuela Reggio Emilia?

\section{CONTEXTO Y PARTICIPANTES}


De manera voluntaria y anónima, se entrevistan a un total de diez profesoras miembros de las escuelas de Reggio Emilia durante una estancia en el marco del programa ERASMUS+ KA101. Las participantes tienen más de diez años de experiencia en la citada metodología y en el sistema educativo italiano. La muestra tiene un carácter intencional y no probabilístico.

\section{PROCESO E INTERPRETACIÓN DE DATOS}

Para la recogida de datos se utiliza la entrevista semi-estructurada grabadas en audio, en lengua italiana, realizadas en los centros escolares de Italia y enfocadas en base a las siguientes dimensiones: metodología, sistema de evaluación, satisfacciones del profesorado sobre su trabajo e importancia del trabajo colaborativo entre la familia y los docentes (Iglesias, Lozano y Sarmiento, 2020). Para el tratamiento de los datos se diseña previamente un mapa de códigos y se emplea el programa cualitativo AQUAD (Huber y Gürtler, 2012) para su codificación final.

\section{RESULTADOS}

El programa cualitativo AQUAD nos ha permitido organizar y codificar la información descrita por los participantes en sus entrevistas. En este sentido se han organizado en 4 temas que pasamos a describir, y en la cuales incluimos ejemplos que corresponden a las narrativas de los participantes.

\subsection{TEMA 1. METODOLOGÍA}

La mayoría de las narrativas de los docentes aseguran estar satisfechos con la metodología Reggio Emilia, la cual se basa en la observación para luego poder ser capaz de crear, se trata de incorporar los medios naturales, hojas, troncos, tierra, agua etc. en todas las actividades posibles, lo que permite que la enseñanza se centre, principalmente, en el aprendizaje del alumnado en contacto continuo con la naturaleza. Los participantes aseguran haber aprendido dicha metodología principalmente en la práctica en las propias escuelas reggianas y en la formación que estas les ofrecen:

\footnotetext{
"La metodología de Reggio Emilia la hemos aprendido aquí mediante la práctica en esta escuela y en otras escuelas de la Basse Reggiana, esta metodología me gusta mucho"

"Yo la aprendí fuera a través de los cursos que imparte la cooperativa, pero lo cierto es que al ser una metodología eminentemente práctica cada día aprendo cosas nuevas y mejoro mi aprendizaje en la escuela"

"Tenemos horas de formación en la propia escuela sobre la metodología Reggio Emilia"
}

Los recursos utilizados son normalmente todos los materiales propios del modelo Reggio Emilia basados principalmente en la naturaleza y todos aquellos que puedan aportar las familias:

"Utilizo los recursos reggianos y todo lo que traigan de casa es bienvenido, sobre todo aquello que sea reciclado o de la naturaleza" 
La ratio docente/alumno es baja, aunque en las aulas se agrupa alumnado de varias edades (tres, cuatro y cinco años) y normalmente rondan los 30 alumnos/as por clase, hay un docente por cada 12 alumnos/as:

"Tengo 29 alumnos pero somos tres docentes"

La planificación se va diseñando poco a poco, se observa las circunstancias y las necesidades de los/as niños/as, así como su curiosidad por ciertos temas para posteriormente plantear las actividades adaptándolas a esas circunstancias. Por otra parte, se diseñan actividades de manera colectiva entre varias escuelas. No hay una base curricular para el diseño de dichas actividades:

\footnotetext{
"Programamos semana por semana según la curiosidad del alumnado y sus necesidades, lo observamos y después diseñamos las actividades"

"Hay numerosas actividades diseñadas de manera colectiva entre varias escuelas, hay una pirámide estructurada para que al final la actividad se trabaje en todas las aulas"

"No existe curriculum"
}

En relación a las adaptaciones de las actividades para alumnado con Necesidades Educativas Específicas no realizan informes de seguimiento, únicamente adaptan según las demandas del alumnado:

\begin{abstract}
"Adaptamos las actividades en base a la circunstancias o al estudiante con dificultades o con mayor capacidad para el aprendizaje, estas adaptaciones no se registran, las actividades se van adaptando de manera espontánea según la necesidad del alumnado"

"Observamos al niño con necesidades y adaptamos la actividad según nuestra propia experiencia, tratamos de cambiar la actividad buscando lo que le pueda ir mejor"
\end{abstract}

\title{
5.2 TEMA 2. SISTEMA EVALUACIÓN
}

Desde las perspectivas tradicionales de la evaluación basadas en un sistema de valoración en el que el estudiante adquiere conocimientos teóricos para mostrarlos en diversas pruebas o exámenes, el pensamiento de los docentes reggianos está orientado más hacia una evaluación formativa basada principalmente en la observación, tal es así que en la Educación Infantil no existe evaluación como tal:

\footnotetext{
"En infantil no evaluamos, cuando el niño llega a cinco años tenemos una entrevista con el director/a y con el profesorado de primaria, hablamos de su capacidad cognitiva, su capacidad de atención, su competencia lingüística..., pero no registramos nada en ningún curso de infantil, tan solo esa entrevista oral"

"Evaluamos mediante la observación, observamos sus capacidades cognitivas y lingüísticas, pero no lo registramos"

"No evaluamos si se han alcanzado los objetivos, no hay un registro evaluativo, hablamos con las familias de sus hijos en general, no de si ha alcanzado un objetivo en concreto"
}

\subsection{TEMA 3. SATISFACCIÓN/INSATISFACCIÓN DOCENTE CON LA PROFESIÓN}


En principio no existen insatisfacciones, los docentes en Reggio Emilia están muy satisfechos con su profesión y con la metodología que imparten. Entre las que más destacan están la oportunidad de trabajar cooperativamente con la comunidad educativa y el amor hacia los niños.

"Lo más bonito de trabajar aquí es cuando los niños te saludan por la calle y te abrazan como parte de su familia"

Otra de las satisfacciones es el reconocimiento social por parte de la Comunidad:

"La comunidad tiene una muy buena imagen de la escuela, solo hay que observar que la matrícula de nuestro alumnado va en aumento"

Por otra parte, otra de las satisfacciones es el aprendizaje a nivel personal que les proporciona la propia metodología.

“A nivel personal la metodología Reggio Emilia me supone un aprendizaje diario"

Sin embargo, existen ciertas dificultades en el desarrollo de esta profesión que los docentes identifican como insatisfacciones y que, especialmente, las relaciones con las compañeras, ya que la metodología Reggio Emilia requiere de una implicación profunda por parte del docente, y eso genera alguna fricción entre ellos:

"Las relaciones, hay gente que no se implica tanto, no es una crítica, pero no hay la misma implicación por parte de todos"

\subsection{TEMA 4. AGENTES QUE INTERVIENEN EN EL PROCESO EDUCATIVO}

El proceso didáctico siempre está unido a la comunicación educativa, la cual está basada, principalmente, en la interacción social de los agentes que intervienen. En este sentido, es altamente beneficioso que docentes y familias actúen de manera colaborativa. De las relaciones entre la familiaescuela se destaca el carácter socializador que puede beneficiar el proceso de enseñanza-aprendizaje. En las escuelas de Reggio Emilia las familias realizan con asiduidad talleres en los que colaboran desarrollando actividades que están normalmente enfocadas a decorar la escuela con la temática que se está trabajando en ese momento en el centro. La familia es importante para las escuelas de la región de Reggio Emilia pero no se llevan a cabo actividades conjuntas en las que participen directamente en el aula, los padres y madres trabajan con las docentes pero no con el alumnado.

"Hay talleres/laboratorios que se organizan por unidades, son encuentros en los que los padres junto a las maestras participan haciendo las actividad que normalmente formaran parte de la decoración de la escuela" 
"Después de los talleres la familia junto a la maestra hace una presentación de lo realizado en el taller y se debate de los temas importantes como las emociones de los propios hijos"

En referencia a las relaciones entre los docentes, hemos observado que los participantes manifiestan que la cooperación entre el profesorado es una estrategia que fomenta el debate flexible y abierto para lograr una coordinación docente basada en el dialogo y que permite adoptar decisiones consensuadas durante el proceso educativo, tal y como hemos identificado en la siguiente narrativa:

\footnotetext{
"Las relaciones entre los docentes son buenas, siempre se tomas decisiones consensuadas y de manera abierta, son decisiones tomadas por todo el colectivo" "Las relaciones con mis colegas son muy buenas trabajamos todas unidas"
}

\section{CONCLUSIONES}

La acción educativa de los docentes reggianos puede ser considerada como ejemplo de buenas prácticas educativas, pues los elementos didácticos, siempre relacionados con la naturaleza, que emplean en sus aulas contribuyen a la calidad de la educación. La metodología se basa principalmente en la observación y experimentación, lo que permite que tanto docentes como alumnado trabajen de manera práctica y vivencial. Se intenta, de este modo, maximizar los potenciales de las habilidades personales de cada alumno, basándose principalmente en la creación tras la observación y la experimentación. Ello es posible gracias a una ratio baja, ya que permite saber mucho de cada uno de los/as alumnos/as, conocer sus habilidades y potenciarlas. Cada escuela se encarga de la contratación del profesorado que necesita adaptándose a las necesidades y particularidades del centro. De ahí que se aprecie, también, un amplio equipo de maestros/as que apueste por esta metodología explotando al máximo sus posibilidades.

A ello se incluyen la no existencia de pruebas estandarizadas y el desarrollo de una evaluación con carácter puramente formativo. De ahí que no se evalúe en la etapa de infantil, y se dé más importancia a informar con todo detalle de las características del alumnado.

Los participantes consideran que la tarea docente llevada a cabo de manera colaborativa junto al resto de sus colegas es sumamente importante para el desarrollo de la profesión, valorando este hecho como una satisfacción profesional.

Se manifiesta una alta satisfacción por parte de los docentes, especialmente, por los beneficios personales y profesionales que implica la metodología Reggio Emilia. Las escasas insatisfacciones son derivadas de la falta implicación de algunos docentes en el quehacer diario.

Las familias es otro de los elementos claves en este proceso, hemos identificado que su participación es continua mediante talleres, pero no hay una intervención directa en el aula.

La colaboración entre los docentes para diseñar la práctica educativa queda patente en sus narraciones. Aunque no describen un proceso reflexivo para la toma de decisiones en su práctica 
educativa, podemos concluir que se detecta un trabajo colaborativo entre el profesorado como estrategia clave en la mejora de la educación, y que además forma parte de la esencia de una comunidad de profesionales de la enseñanza que se enriquece gracias a las aportaciones de cada docente.

Nuestra principal conclusión es que la metodología desarrollada en las escuelas de Reggio Emilia aportan a los participantes de programa ERASMUS+ un modelo de saber profesional basado en las buenas prácticas didácticas, pero en especial de compromiso con la educación y el aprendizaje del alumnado. En este sentido el programa ERASMUS+ puede considerarse como modelo de formación docente que contribuye a la calidad de la enseñanza y del aprendizaje, por la implementación en otros contextos escolares, tal y como hemos mostrado en esta comunicación. 


\section{REFERENCIAS}

Arroyo, S., Iglesias, M. J., y Lozano, I. (2021). Los programas de movilidad estudiantil en la Educación Superior: el caso de una universidad española. South Florida Journal of Development, 2 (2), 1474-1488. DOI: $10.46932 /$ sfjdv2n2-028

Atkins, L., y Wallace, S. (2012). Qualitative Research in Education. Sage: London.

Blanco, L., Iglesias, M. J., y Lozano, I. (2017). The ERASMUS+ programme for teacher educational innovation: visiting Finland. Paper in 10th annual International Conference of Education, Research and Innovation 16-18 November,

Seville, Spain.Bonell, L., y Ríos, O. (2014). Participation and family education in school: Successful educational actions. Studies in the Education of Adults, 46(2), 177-191,

Carins, D., Kraklewska, E., Cuzzocrea, V., y Allaste, A-A, (2018). Mobility, Education and Employatibility in the European Union. Inside Erasmus. Lisboa: Palgrave Macmillan.

Dotger, B. H. (2015). Core Pedagogy: Individual Uncertainty, Shared Practice, Formative Ethos. Journal of Teacher Education, 66 (3), 215-226.

Dwyer, R., y Emerald, E. (2017). Narrative Research in Practice: Navigating the Terrain. En Dwyer, R., Davis, I., Emerald, E. (eds) Narrative Research in Practice (pp. 1-25). Singapore: Springer.

Giner, A., Iglesias, M. J., Lozano, I., y Urrea, M. E. (2017). Innovación docente en la universidad: el caso de la implementación del contenido "Comunidades de Aprendizaje" en dos asignaturas en la formación inicial del profesorado. En R. Roig-Vila (coord.). Investigación en docencia universitaria: Diseñando el futuro a partir de la innovación educativa (pp. 538-547). Barcelona: Octaedro.

Goodson, I. Antikainen, A., Sikes, P., y Andrews, M. (edit) (2016). The Routledge International Handbook on Narrative and Life History. New York: Routledge.

European Commission. (2016). Erasmus+ programme guide. Luxembourg: Publications Office of the European Union.

European Commission. (2017). Erasmus + programme guide version 2. Luxembourg: Publications Office of the European Union.

Flores, M. A. (2017). Practice, theory and research in initial teacher education: international perspectives. European Journal of Teacher Education, 40(3) 287-290.

Huber, G. L., y Gürtler, L. (2012). AQUAD. Manual del programa para analizar datos cualitativos (1. ed. 2003, Tübingen: Ingeborg Huber Verlag). Tübingen: Günter Huber.

Iglesias, M. J., Lozano, I., y Roldán, I. (2018). La calidad e innovación educativa en la formación continua docente: un estudio cualitativo en dos centros educativos. Revista Iberoamericana de Educación, 77(1), 13-34. 
Iglesias, M. J., Lozano, I., y Sarmiento, M. (2020). Las acciones didácticas del profesorado novel universitario: un estudio cualitativo desde una perspectiva interdisciplinar. Revista Paradigmas SocioHumanísticos, 2(1): 26-41. https://doi.org/10.26752/revistaparadigmassh.v1i2.468

Opfer, V. D., y Pedder, D. (2011). Conceptualizing teacher professional learning. Review of Educational Research, 81 (3) 376-407.

Vaillant, D. (2016). Trabajo colaborativo y nuevos escenarios para el desarrollo profesional docente. Docencia, 60, 5-13. 